\title{
Simulations of structure formation in interacting dark energy cosmologies
}

\author{
M. Baldi ab*

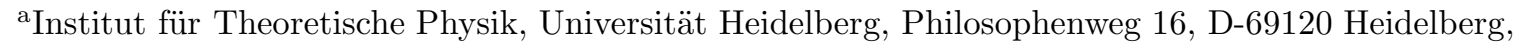 \\ Germany. \\ ${ }^{\mathrm{b}}$ Max-Planck-Institut für Astrophysik, Karl-Schwarzschild Strasse 1, D-85748 Garching, Germany.
}

The evidence in favor of a dark energy component dominating the Universe, and driving its presently accelerated expansion, has progressively grown during the last decade of cosmological observations. If this dark energy is given by a dynamic scalar field, it may also have a direct interaction with other matter fields in the Universe, in particular with cold dark matter. Such interaction would imprint new features on the cosmological background evolution as well as on the growth of cosmic structure, like an additional long-range fifth-force between massive particles, or a variation in time of the dark matter particle mass. We review here the implementation of these new physical effects in the N-body code GADGET-2, and we discuss the outcomes of a series of high-resolution N-body simulations for a selected family of interacting dark energy models, as already presented in Baldi et al. 20]. We interestingly find, in contrast with previous claims, that the inner overdensity of dark matter halos decreases in these models with respect to $\Lambda \mathrm{CDM}$, and consistently halo concentrations show a progressive reduction for increasing couplings. Furthermore, the coupling induces a bias in the overdensities of cold dark matter and baryons that determines a decrease of the halo baryon fraction below its cosmological value. These results go in the direction of alleviating tensions between astrophysical observations and the predictions of the $\Lambda$ CDM model on small scales, thereby opening new room for coupled dark energy models as an alternative to the cosmological constant.

\section{INTRODUCTION}

The existence of dark energy (DE) and cold dark matter (CDM) as the two most abundant components of the Universe keeps acquiring support as new and more detailed observational data become available. Throughout the last decade, a large number of independent and complementary datasets (e.g. [1, 2, 3, 4, 5]) have shown that the Universe is almost spatially flat, with a total matter density of at most $24 \%$ of the critical density, and the remaining $76 \%$ being made of a DE component able to drive an accelerated expansion. The nature of this dominant component, however, is still unknown and its understanding constitutes a serious theoretical challenge. Dynamical DE models based on the evolution of a self-interacting scalar field have been proposed [6, 7] as a possible way to overcome the severe fine-tuning problems that affect the (otherwise very successful) cosmological constant. An interesting idea in the context of dynamical DE models is then given by the recently suggested possibility of a direct interaction between the dark energy scalar field and other matter species in the Universe [8, 9, 10, 11, 12]. Understanding in detail what effects this interaction imprints on observable features [13, 14, 15, 16, 17, 18, 19] is therefore a crucial step for a direct observational test of these models.

In this paper, after briefly recalling the essential features of interacting DE models and discussing their implementation in numerical Nbody codes, we present the results of the first fully self-consistent high-resolution hydrodynamic simulations of cosmic structure formation for a selected family of interacting DE cosmologies.

The results presented here are discussed in larger detail and in a more complete fashion in 20].

*mbaldi@mpa-garching.mpg.de 


\section{INTERACTING DARK ENERGY COSMOLOGIES}

Interacting dark energy cosmologies can be described as a multicomponent system where, although the conservation of the total stress energy tensor $T^{\mu}{ }_{\nu}$ is not violated:

$\nabla_{\mu} T_{\nu}^{\mu}=\sum_{\alpha} \nabla_{\mu} T_{(\alpha) \nu}^{\mu}=0$,

the individual stress energy tensor $T^{\mu}{ }_{\nu(\alpha)}$ of each species $\alpha$ is in general not conserved. If so, its divergence has a source term $Q_{(\alpha) \nu}$ accounting for the coupling between the individual species:

$\nabla_{\mu} T_{(\alpha) \nu}^{\mu}=Q_{(\alpha) \nu}$,

and the conservation of the total stress energy tensor (Eqn. 1) translates into the constraint

$\sum_{\alpha} Q_{(\alpha) \nu}=0$.

We consider then a system described by the Lagrangian:

$\mathcal{L}=-\frac{1}{2} \partial^{\mu} \phi \partial_{\mu} \phi-U(\phi)-m(\phi) \bar{\psi} \psi+\mathcal{L}_{\text {kin }}[\psi]$,

where the role of the $\mathrm{DE}$ is played by a scalar field $\phi$ with self-interaction potential $U(\phi)$, and where the mass of matter fields $\psi$ coupled to the $\mathrm{DE}$ is also a function of $\phi$. The choice $m(\phi)$ then specifies the source term $Q_{(\phi) \nu}$ via the expression:

$Q_{(\phi) \nu}=\frac{\partial \ln m(\phi)}{\partial \phi} \rho_{c} \partial_{\nu} \phi$.

Due to the constraint (3), if DE couples only to cold dark matter (CDM, hereafter denoted with a subscript $c$ ) one has that $Q_{(c) \nu}=-Q_{(\phi) \nu}$. In the following we will assume that the latter condition always holds. In particular, in a cosmological system composed by DE, CDM, and baryons, we will always assume the baryons (denoted with a subscript $b$ ) to have no coupling to the DE.

The zero-component of equation (21) provides the conservation equations for the energy densities of each species:

$$
\begin{aligned}
\rho_{\phi}^{\prime} & =-3 \mathcal{H} \rho_{\phi}\left(1+w_{\phi}\right)-Q_{(\phi) 0}, \\
\rho_{c}^{\prime} & =-3 \mathcal{H} \rho_{c}+Q_{(\phi) 0}, \\
\rho_{b}^{\prime} & =-3 \mathcal{H} \rho_{b},
\end{aligned}
$$

which will determine the background evolution of the Universe. Here a prime denotes a derivative with respect to the conformal time $\tau$, defined via $d \tau \equiv d t / a$, and $\mathcal{H}=a^{\prime} / a$ is the conformal Hubble function, while $w_{\phi} \equiv p_{\phi} / \rho_{\phi}$ is the equation of state of the DE.

We will focus our attention here on a class of models identified by the choice:

$m(\phi)=m_{0} e^{-\beta_{c}(\phi) \frac{\phi}{M}} \Rightarrow Q_{(\phi) 0}=-\frac{\beta_{c}(\phi)}{M} \rho_{c} \phi^{\prime},(9$

where $M \equiv 1 / \sqrt{8 \pi G_{N}}$ and $G_{N}$ is Newton's gravitational constant.

This set of cosmologies has been widely investigated, for the case of a constant coupling function, with regard to its background and linear perturbation features [8, 9], to its effects on structure formation [21, 22], and also via a first N-body simulation [23].

By perturbing up to linear order the full system of equations governing our cosmological framework it is possible to derive an effective acceleration equation for a CDM test particle at a distance $r$ from another CDM particle of mass $\tilde{M}_{c}$, which takes the form (for a complete and rather compact derivation see [20]):

$\dot{\vec{v}}_{c}=-H\left(1-\frac{\beta_{c}(\phi)}{M} \frac{\dot{\phi}}{H}\right) \vec{v}_{c}-\vec{\nabla} \frac{\tilde{G}_{c} \tilde{M}_{c}}{r}$.

This equation is manifestly different in three aspects from the usual newtonian acceleration equation in an expanding Universe: first, the velocitydependent term now contains an additional contribution proportional to the DE-CDM coupling $\beta_{c}(\phi)$; second, the CDM test particle feels an effective gravitational constant $\tilde{G}_{c}$ given by [21]:

$\tilde{G}_{c} \equiv G_{N}\left[1+2 \beta_{c}^{2}(\phi)\right] ;$

third, as a consequence of the energy exchange with the DE scalar field (Eqn. 7), the mass $\tilde{M}_{c}$ of the CDM particle sourcing the gravitational potential changes in time according to the expression:

$\tilde{M}_{c} \equiv M_{c} e^{-\int \beta_{c}(\phi) \frac{\mathrm{d} \phi}{\mathrm{d} a} \mathrm{~d} a}$.

In the N-body analysis described in the following, we consider $\beta_{c}(\phi)$ to be constant, so that the effective mass formally reads $\tilde{M}_{c} \equiv M_{c} e^{-\beta_{c}\left(\phi-\phi_{0}\right)}$. 
Eqn. [10 is very important for our discussion since it represents the starting point for the implementation of coupled DE models in an N-body code. It is essential to stress here the vectorial nature of Eqn. 10, which is a key point to understand the effects of the new physics induced by the DE-CDM interaction and hence needs to be properly taken into account for a correct implementation in N-body algorithms.

\section{THE SIMULATIONS}

The main aim of our investigation is to explore the effects that arise in the process of cosmic structure formation as a consequence of the interaction between the DE scalar field and the CDM fluid. Our focus will concentrate on the nonlinear regime of structure formation, and to this end we study a set of high-resolution cosmological N-body simulations performed with the code GADGET-2 24], that we suitably modified for this purpose. Previous attempts to use cosmological N-body simulations for different flavours of modified Newtonian gravity have been discussed, for instance, in [23, 25, 26, 27, 28, 29, 30, 31, 32] but to our knowledge [23] is the only previous work focusing on the properties of nonlinear structures in models of coupled quintessence. For this reason we apply our methodology to a series of interacting DE models identical to the ones considered in [23], where the only difference with respect to this previous work amounts to updating the cosmological parameters to the latest WMAP [2] results $\left(\Omega_{c}=0.213, \Omega_{b}=0.044\right.$, $\left.\Omega_{D E}=0.743, \sigma_{8}=0.769, h=0.719, n=0.963\right)$. In these models the scalar field $\phi$ has a RatraPeebles [7] self-interaction potential

$U(\phi)=\frac{\Lambda^{4+\alpha}}{\phi^{\alpha}}$,

with a slope $\alpha=0.143$, and with a constant coupling $\beta_{c}$ to CDM particles only, as described above; we label them as $\mathrm{RP} n$ for values of the coupling $\beta_{c}=n \times 0.04$, with $n$ ranging from 1 to 5 , in analogy with [23].

For all the models - and for a reference $\Lambda$ CDM cosmology with the same cosmological parameters - we run low-resolution simulations in a large box $\left(L_{\text {box }}=320 h^{-1} \mathrm{Mpc}, N_{\text {part }}=2 \times 128^{3}\right)$ to test the large-scale behaviour of the different cosmologies and the convergence of our implementation; for four of these models ( $\Lambda$ CDM, RP1, RP2, RP5) we then run high-resolution simulations in a smaller box $\left(L_{\mathrm{box}}=80 h^{-1} \mathrm{Mpc}\right.$, $N_{\text {part }}=2 \times 512^{3}$ ) to investigate the properties of highly nonlinear structures.

\subsection{Methods}

We now describe one by one the main distinctive features of our models and their implementation in the N-body code GADGET-2. For a more detailed description of our numerical approach see [20].

Modified expansion rate - The interaction between DE and CDM modifies the background evolution through the presence of an early DE component during all the period of structure formation. The effect of such early DE is to modify the expansion history of the Universe. For the same set of cosmological parameters at $z=0$, different values of the coupling $\beta_{c}$ will then lead to different expansion histories that will have to be separately computed and then used for the N-body time integration.

Mass variation - As discussed above, the coupled species (CDM in our case) feature an effective particle mass that changes in time according to Eqn. 12 Therefore, the mass of CDM particles within the simulation box will have to be accordingly corrected at each timestep, while baryon particles will keep a constant mass.

Velocity-dependent acceleration - As shown in Eqn. 10, the coupling induces an additional velocitydependent term in the acceleration equation for CDM particles. Therefore, an additional term of the form

$\vec{a}_{v} \equiv \beta_{c} \frac{\dot{\phi}}{M} \vec{v}$

has to be explicitely added to the acceleration of every CDM particle at each timestep.

Fifth-force - Finally, one of the most important modifications introduced by the DE-CDM interaction is the presence of a long-range fifth-force effectively represented by a modified gravitational constant, formally written as in Eqn. 11] for the gravitational interaction of two CDM particles, while any interaction involving a baryon particle will be governed by the standard gravitational constant $G_{N}$. This dependence of the gravitational strength on the type of the particles involved in the interaction requires an $\mathrm{N}$-body code to be able to distinguish among differ- 
ent particle types in the gravitational force calculation. In GADGET-2, the gravitational interaction is computed by means of a TreePM hybrid method (see 24] for details about the TreePM algorithm), so that both the tree and the particle-mesh algorithms have been modified in order to account for this effectively species-dependent gravity.

\subsection{Tests}

As a test of our implementation we check whether the linear growth of density fluctuations in the simulations is in agreement with the linear theory prediction for each coupled DE model under investigation. To do so, we compute the growth factor from the lowresolution simulations and we compare it with the solution of the system of equations for the evolution of linear perturbations in interacting DE models, numerically integrated with a suitably modified version of the Boltzmann code CMBEASY 33]. The comparison is shown in the left panel of Fig. 1. The accuracy of the linear growth computed from the simulations in fitting the theoretical prediction is of the same order for all the values of the coupling, and the discrepancy within the two never exceeds a few percent.

\section{RESULTS}

We present here the most relevant outcomes of the high-resolution simulations of coupled DE cosmologies described above.

As first basic analysis steps we apply the Friendsof-Friends (FoF) and SUBFIND algorithms 34 to identify groups and gravitationally bound subgroups in each of our simulations. Given that the seed used for the random realization of the power spectrum in the initial conditions is the same for all the different runs, structures will form roughly at the same positions in all simulations. Therefore it is possible, in general, to identify the same objects in all the simulations and to directly compare their properties. We restrict the comparative analysis of our simulations to the 200 most massive halos identified by the FoF algorithm, which have virial masses ranging from $4.64 \times 10^{12} h^{-1} M_{\odot}$ to $2.83 \times 10^{14} h^{-1} M_{\odot}$.

\subsection{Halo density profiles}

With a suitable procedure we can select, among the 200 most massive halos found at $z=0$ in each of our simulations, those objects that can be identified with certainty as being the same structure emerging in the different cosmologies. This leaves 74 objects for our comparison analysis. For these halos we com- pute the spherically averaged density profiles of CDM and baryons as a function of radius around the position of the particle with the minimum gravitational potential.

Interestingly, the halos formed in the coupled DE cosmologies show systematically a lower inner overdensity with respect to $\Lambda \mathrm{CDM}$, and this effect grows with increasing coupling. This is clearly visible in the right panel of Fig. 1 where we show the density profiles of CDM and baryons for the most massive halo in our sample in the four cosmologies with different couplings for which we have high-resolution simulations. We remark that this result is clearly incompatible with the essentially opposite behaviour previously reported by [23].

In fact, unlike found in [23], all the halos in our comparison sample have density profiles that are well fit by the NFW fitting function [35]

$$
\left[\frac{\rho(r)}{\rho_{\text {crit }}}\right]_{\mathrm{NFW}}=\frac{\delta^{*}}{\left(r / r_{s}\right)\left(1+r / r_{s}\right)^{2}},
$$

independent of the value of the coupling. The scale radius $r_{s}$ increases for each halo with increasing coupling $\beta_{c}$, and becomes larger than that found in $\Lambda$ CDM. Therefore, the coupling does not affect the overall shape of the density profiles, but rather the transition point between the two asymptotic slopes of the NFW function. In other words, the halos become less concentrated with increasing coupling.

\subsection{Halo concentrations}

For all the 200 most massive halos found in each of our high-resolution simulations we compute halo concentrations as $c=r_{200} / r_{s}$, based on our NFW fits to the halo density profiles. Here $r_{200}$ is the radius enclosing a mean overdensity 200 times the critical density.

Consistently with the trend found for the inner overdensity in the halo density profiles, we find that halo concentrations are on average significantly lower for coupled DE models with respect to $\Lambda \mathrm{CDM}$, and the effect again increases with increasing coupling $\beta_{c}$. This behaviour is shown explicitly in the left panel of Fig. 2, where we plot halo concentrations as a function of the halo virial mass $M_{200}$ for our four highresolution simulations.

It is possible to show (see [20] for details) that this effect is not due to a change in the formation epoch of structures in the coupled cosmologies with respect to $\Lambda$ CDM. Indeed, a more detailed investigation of this effect shows that the decrease of concentrations arises as a consequence of the expansion of virialized 

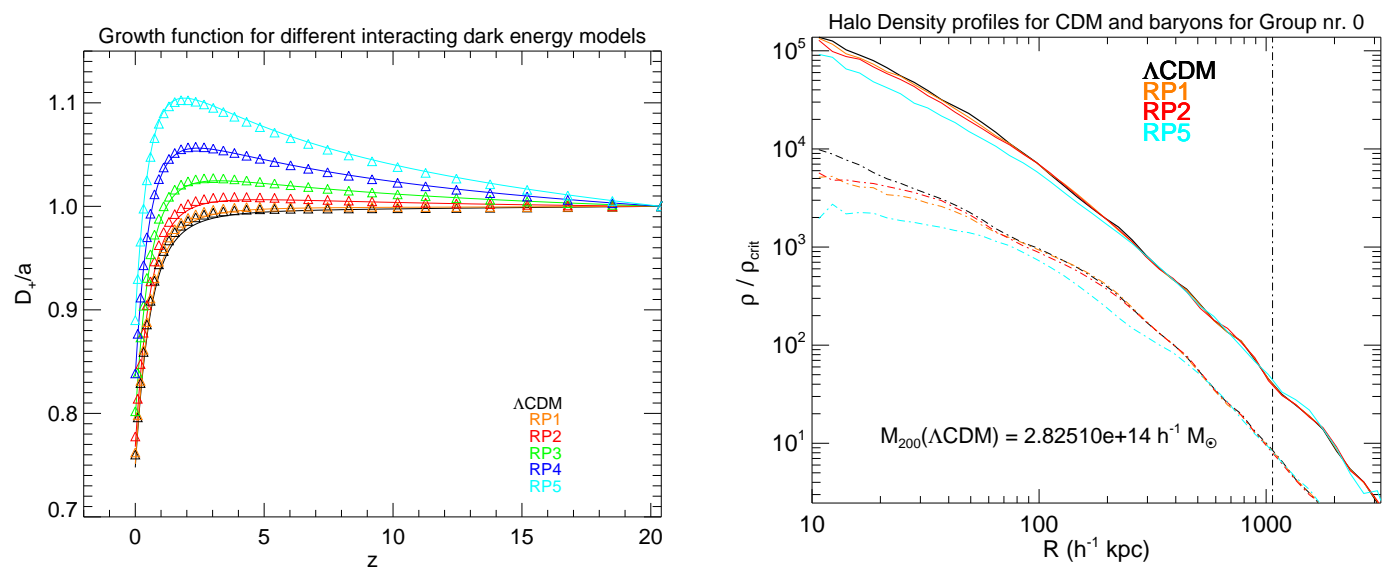

Figure 1. Left Panel: Evolution of the growth function with redshift for the models of coupled DE investigated with the low-resolution simulations. The solid lines are the total growth functions as evaluated numerically with CMBEASY, while the triangles are the growth function evaluated from the simulations. Right Panel: Density profiles of CDM (solid lines) and baryons (dot-dashed lines) for the most massive halo in the simulation box at $z=0$. The vertical dot-dashed line indicates the location of the virial radius for the $\Lambda \mathrm{CDM}$ halo.

structures. This expansion is a direct consequence of the DE-CDM interaction, and can be explained as follows: on one side, the potential wells of CDM halos become shallower as time goes by as a consequence of the mass decrease of their CDM content, as described by Eqn. 12 on the other side, the new velocity-dependent acceleration (Eqn. 14) speeds up the motion of CDM particles, thereby injecting energy into the system. The combination of these two effects determines an overall increase of the total energy of the system, which will then slightly expand to restore virial equilibrium.

It is interesting to note that the effects we find go in the direction of less "cuspyness" of halo density profiles, which is preferred by observations and thus in fact opens up new room for the phenomenology of interacting DE models.

\subsection{Halo baryon fraction}

The extra force proportional to $\beta_{c}^{2}$ (see Eqn. 11) that affects only the interaction between two CDM particles induces a bias in the evolution of density fluctuations of baryons and CDM [36, 37, 38]. This bias will appear at all scales during the period of linear growth of density fluctuations, which makes it clearly distinguishable from the hydrodynamic bias arising only at small scales as structure evolves. Furthermore, as it was shown in 23] and [20], this grav- itational bias is enhanced in the nonlinear stages of gravitational clustering, and reaches its largest amplitude in the core of highly overdense regions.

It is interesting that the above effect produces a baryon deficit in virialized halos, i.e. they contain fewer baryons than expected based on their mass and the universal cosmological baryon fraction. In particular, this means that one can not expect that baryon fractions determined through X-ray measurements in clusters would yield the cosmological value. In order to give a rough estimate of the magnitude of the discrepancy we compute the relative baryon fraction within the virial radius $r_{200}$ of all the 200 halos in our sample, defined as

$Y_{b} \equiv \frac{f_{b}}{\Omega_{b} / \Omega_{m}} \quad$ where $\quad f_{b} \equiv \frac{M_{b}\left(<r_{200}\right)}{M_{\mathrm{tot}}\left(<r_{200}\right)}$.

For the $\Lambda \mathrm{CDM}$ case, our results for the evolution of $Y_{b}$ are consistent with the value of $Y_{b} \sim 0.92$ found by the Santa Barbara Cluster Comparison Project 39], and with the more recent results of [40] and 41], while for the coupled models the relative baryon fraction shows a progressive decrease with increasing coupling, down to a value of $Y_{b} \sim 0.86-0.87$ for the RP5 case, as shown in the right panel of Fig. 2 .

It is also important to notice that this effect is always towards lower baryon fractions in clusters with respect to the cosmological value. This could in fact 

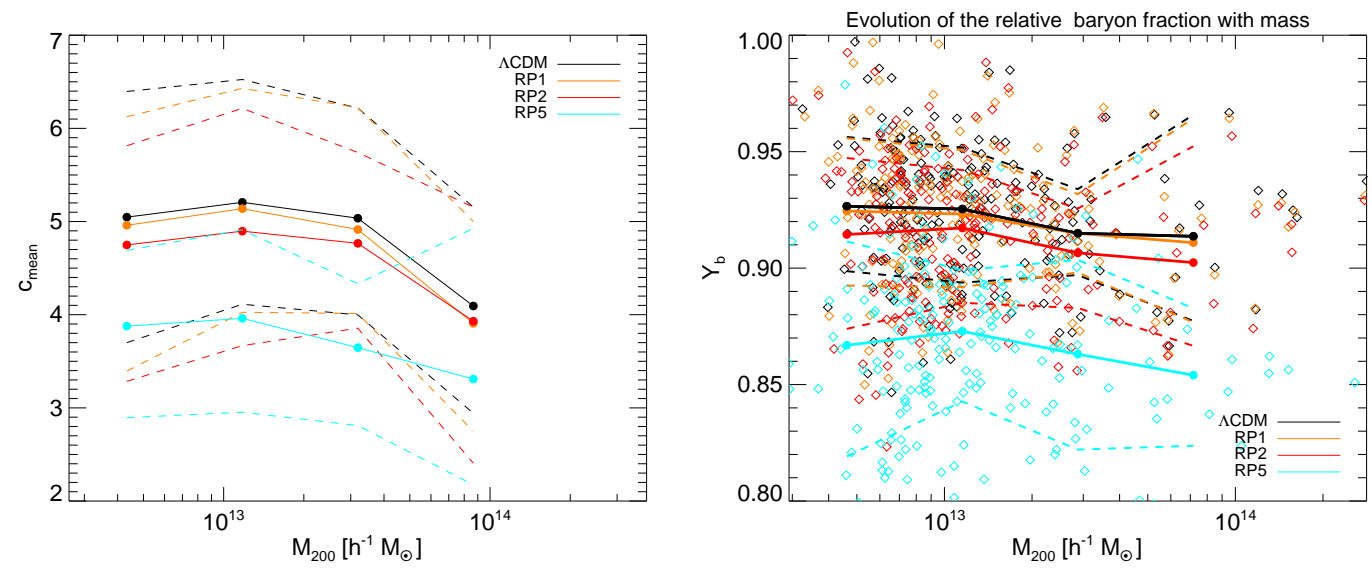

Figure 2. Left Panel: Evolution of the mean halo concentration as a function of mass for the 200 most massive halos in our simulations and for the different cosmological models under investigation. The halos have been binned by mass, and the mean concentration in each bin is plotted as a filled circle. Right Panel: Evolution with virial mass $M_{200}$ of the relative baryon fraction $Y_{b}$ within the virial radius $r_{200}$ for all the halos in our sample. The coloured diamonds represent the relative baryon fraction of each single halo, while the filled circles and the coloured curves show the behaviour of the mean relative baryon fraction in each mass bin for our four high-resolution simulations.

alleviate tensions between the high baryon abundance estimated from CMB observations, and the somewhat lower values inferred from detailed X-ray observations of galaxy clusters [42, 43, 44].

\section{CONCLUSIONS}

We have investigated the effects that arise in the nonlinear regime of structure formation in the context of interacting DE models, by means of detailed high-resolution N-body simulations run with a suitably modified version of the code GADGET-2. The numerical implementation we have developed is quite general and not restricted to the simple specific models of coupled quintessence that we have discussed in this work. Instead, it should be well suited for a much wider range of $\mathrm{DE}$ models.

We have presented here the main couclusions of our analysis, concerning the properties of collapsed structures in interacting DE models. In particular, we have shown that CDM halo density profiles are remarkably well fit over the resolved range by the NFW formula for any value of the coupling, but there is a clear trend of a decrease of the inner halo overdensity with respect to $\Lambda \mathrm{CDM}$ with increasing coupling (or, equivalently, an increase of the scale radius $r_{s}$ for increasing coupling). This result conflicts with previous claims for the same class of coupled DE models 23]. Consistently, also halo concentrations are reduced with increasing coupling with respect to $\Lambda \mathrm{CDM}$.

Finally, as already shown in [23], we also find that, as a consequence of the different effective gravitational strength between CDM and baryons, these two components develop a bias in the amplitude of their relative density fluctuations, and this bias is enhanced in the nonlinear region within and around massive halos. The enhancement of the bias in highly nonlinear structures has an impact on the determination of the baryon fraction from cluster measurements, and we have computed for all our halos its evolution with coupling, finding that the baryon fraction is reduced with increasing coupling by up to $\sim 8-10 \%$ with respect to $\Lambda \mathrm{CDM}$ for the largest coupling value we consider.

\section{ACKNOWLEDGMENTS}

I would like to thank the organizers of this Dark Energy Conference for the opportunity of presenting these results and for the very nice atmosphere of the meeting. I also want to acknowledge V. Pettorino, G. Robbers, and V. Springel for their collaboration to the work presented in this contribution. This work has been supported by the TRR33 Transregio Collab- 
orative Research Network on the "Dark Universe", and by the DFG Cluster of Excellence "Origin and Structure of the Universe".

\section{References}

[1] The 2dFGRS, W.J. Percival et al., Mon. Not. Roy. Astron. Soc. 327 (2001) 1297, astro-ph/0105252.

[2] WMAP, E. Komatsu et al., (2008), 0803.0547.

[3] Supernova Search Team, A.G. Riess et al., Astron. J. 116 (1998) 1009, astro-ph/9805201.

[4] Supernova Cosmology Project, S. Perlmutter et al., Astrophys. J. 517 (1999) 565, astro-ph/9812133.

[5] The SNLS, P. Astier et al., Astron. Astrophys. 447 (2006) 31, astro-ph/0510447.

[6] C. Wetterich, Nucl. Phys. B302 (1988) 668.

[7] B. Ratra and P.J.E. Peebles, Phys. Rev. D37 (1988) 3406 .

[8] C. Wetterich, Astron. Astrophys. 301 (1995) 321, hep-th/9408025.

[9] L. Amendola, Phys. Rev. D62 (2000) 043511, astroph/9908023.

[10] G.R. Farrar and P.J.E. Peebles, apj 604 (2004) 1, arXiv:astro-ph/0307316.

[11] S.S. Gubser and P.J.E. Peebles, prd 70 (2004) 123511, arXiv:hep-th/0407097.

[12] G.R. Farrar and R.A. Rosen, Physical Review Letters 98 (2007) 171302, arXiv:astro-ph/0610298.

[13] R. Bean et al., (2008), 0808.1105.

[14] O. Bertolami, F. Gil Pedro and M. Le Delliou, Phys. Lett. B654 (2007) 165, astro-ph/0703462.

[15] S. Matarrese, M. Pietroni and C. Schimd, JCAP 0308 (2003) 005, astro-ph/0305224.

[16] B. Wang et al., Nucl. Phys. B778 (2007) 69, astro$\mathrm{ph} / 0607126$.

[17] Z.K. Guo, N. Ohta and S. Tsujikawa, Phys. Rev. D76 (2007) 023508, astro-ph/0702015.

[18] R. Mainini and S. Bonometto, JCAP 0706 (2007) 020, astro-ph/0703303.

[19] S. Lee, G.C. Liu and K.W. Ng, Phys. Rev. D73 (2006) 083516, astro-ph/0601333.

[20] M. Baldi et al., (2008), 0812.3901.

[21] L. Amendola, Phys. Rev. D69 (2004) 103524, astro$\mathrm{ph} / 0311175$.
[22] V. Pettorino and C. Baccigalupi, Phys. Rev. D77 (2008) 103003, 0802.1086.

[23] A.V. Macciò et al., Phys. Rev. D69 (2004) 123516, astro-ph/0309671.

[24] V. Springel, Mon. Not. Roy. Astron. Soc. 364 (2005) 1105 , astro-ph/0505010.

[25] A. Nusser, S.S. Gubser and P.J.E. Peebles, Phys. Rev. D71 (2005) 083505, astro-ph/0412586.

[26] H.F. Stabenau and B. Jain, Phys. Rev. D74 (2006) 084007, astro-ph/0604038.

[27] M. Kesden and M. Kamionkowski, Phys. Rev. D74 (2006) 083007, astro-ph/0608095.

[28] V. Springel and G.R. Farrar, MNRAS380 (2007) 911, arXiv:astro-ph/0703232.

[29] I. Laszlo and R. Bean, Phys. Rev. D77 (2008) 024048, 0709.0307.

[30] P.M. Sutter and P.M. Ricker, (2008), 0804.4172.

[31] H. Oyaizu, (2008), 0807.2449.

[32] J.A. Keselman, A. Nusser and P.J.E. Peebles, (2009), 0902.3452

[33] M. Doran, JCAP 0510 (2005) 011, astro-ph/0302138.

[34] V. Springel et al., MNRAS328 (2001) 726, arXiv:astro-ph/0012055.

[35] J.F. Navarro, C.S. Frenk and S.D.M. White, Astrophys. J. 490 (1997) 493, astro-ph/9611107.

[36] R. Mainini, Phys. Rev. D72 (2005) 083514, astro$\mathrm{ph} / 0509318$.

[37] R. Mainini and S. Bonometto, Phys. Rev. D74 (2006) 043504, astro-ph/0605621.

[38] M. Manera and D.F. Mota, Mon. Not. Roy. Astron. Soc. 371 (2006) 1373, astro-ph/0504519.

[39] C.S. Frenk et al., ApJ525 (1999) 554, arXiv:astro$\mathrm{ph} / 9906160$.

[40] S. Ettori et al., Mon. Not. Roy. Astron. Soc. 365 (2006) 1021, astro-ph/0509024.

[41] S. Gottloeber and G. Yepes, Astrophys. J. 664 (2007) 117, astro-ph/0703164.

[42] A. Vikhlinin et al., Astrophys. J. 640 (2006) 691, astro-ph/0507092.

[43] I.G. McCarthy, R.G. Bower and M.L. Balogh, Mon. Not. Roy. Astron. Soc. 377 (2007) 1457, astro$\mathrm{ph} / 0609314$.

[44] S. LaRoque et al., Astrophys. J. 652 (2006) 917, astro-ph/0604039. 